\title{
Lombalgia e o equilíbrio corporal de atletas da seleção brasileira feminina de canoagem velocidade
}

\author{
Low back pain and corporal balance of female brazilian selection \\ canoeing flatwater athletes
}

\author{
Luiz Fernando Cuozzo Lemos \\ Clarissa Stefani Teixeira \\ Carlos Bolli Mota
}

1 Universidade Federal de Santa Maria. Laboratório de Biomecânica. Santa Maria, RS. Brasil

2 Universidade Federal de Santa Catarina. Doutoranda em Engenharia de Produção. Florianópolis, SC. Brasil

Recebido em 27/05/09 Revisado em 13/09/09 Aprovado em 31/03/10

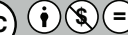

Resumo - A dor lombar vem sendo apontada como interveniente na estabilidade corporal. No esporte, o estudo dessas relações é importante visto que os acometimentos podem influenciar os resultados dos treinamentos e das competições. Em mulheres canoístas de elite, a literatura reporta altos índices de lesões na região lombar. $\mathrm{O}$ estudo teve como objetivo verificar a influência da dor lombar sobre o equilíbrio corporal de atletas da seleção brasileira feminina de canoagem velocidade. Métodos: As atletas com dor lombar (na última semana) foram comparadas com as atletas sem dor, em condições bipodal e unipodal, com olhos abertos e fechados, utilizando-se uma plataforma de força AccuSway Plus, da marca AMTI (Advanced Mechanical Technology, INC). As variáveis analisadas foram a amplitude do centro de pressão na direção ântero-posterior (COPap) e na direção médio-lateral (COPml). Os resultados mostraram maior instabilidade corporal para as atletas com queixas de dor lombar. No COPml apenas não apresentaram diferença estatisticamente significativa uma das condições $(p=0,901)$ e o COPap apenas duas $(p=0,414$ e p=0,567), ambas em tentativas unipodais e de olhos fechados. Pode-se concluir que a dor na região lombar indicou ser prejudicial ao equilíbrio corporal de canoístas de alto nível do gênero feminino.

Palavras-chave: Equilíbrio postural; Atletas; Dor lombar.

Abstract - Low back pain has been shown to interfere with body stability. In sports, the study of these relationships is important because the bouts may influence the results of training and competitions. High rates of injury to the lumbar region have been reported for elite female canoe paddlers. The objective of this study was to assess the influence of low back pain on body balance in female athletes of the Brazilian flatwater canoe team. Athletes with low back pain (in the last week) were compared to those without pain in the two-leg and single-leg stance, with the eyes open and closed, using an AccuSway Plus force platform (Advanced Mechanical Technology, Inc.). The variables analyzed were the amplitude of the pressure center in the anteroposterior direction (COPap) and medial-lateral direction (COPml). The results showed higher body instability for athletes with low back pain. For COPml, no significant difference between the two groups was observed for only one condition (single-leg stance) $(p=0.901)$, and for COPap, no significant difference was observed for two conditions ( $p=0.414$ and $p=0.567)$, i.e., single-leg stance and eyes closed. In conclusion, low back pain was harmful to body balance in high-level female canoe paddlers.

Key words: Postural balance; Athletes; Low back pain. 


\section{INTRODUÇÃO}

A modalidade de canoagem passou a ser esporte olímpico no ano de 1936, em Berlin ${ }^{1}$, o que provocou desenvolvimento de métodos específicos de treinamento do esporte e uma técnica que possibilitasse o melhor desempenho com menor gasto energético. A melhor técnica para o deslocamento da embarcação necessita de torção de tronco do atleta $^{2}$, com movimentos repetitivos de forma cíclica $^{3}$, realizada na posição sentada, com o quadril em flexão. Segundo Medrado ${ }^{4}$, estes movimentos, realizados de forma repetitiva, geram propensão a hérnia lombar. De forma geral, a dor lombar atinge cerca de $45 \%$ da população durante seu período de vida produtivo, sendo que, para Leveille et al. ${ }^{5}$ e Andersen et al. ${ }^{6}$, as mulheres apresentam maior susceptibilidade a esse quadro clínico.

De forma geral, as lesões esportivas causam alterações no desempenho dos atletas, com um possível afastamento dos treinos e competições ${ }^{3}$. A realização de movimentos repetitivos por períodos de tempos prolongados, durante o treinamento, normalmente realizado em dois turnos diários, totalizando de seis a oito horas, não é considerada a única problemática da modalidade? . A posição dentro da embarcação, considerada como não sendo ergonomicamente correta, é um agravante para possíveis lombalgias em canoístas e estas considerações agravam-se pela flexão somada às rotações de tronco necessárias para o desenvolvimento do esporte.

Panjabi ${ }^{8}$ define o mecanismo que influencia a dor lombar na postura como a perda da habilidade da coluna em suportar cargas fisiológicas impostas a ela, principalmente, durante a manutenção de más posturas por tempo prolongado como, por exemplo, na canoagem, não havendo deficiência neurológica, nem deformidade. $\mathrm{O}$ mesmo autor expõe que a dor nessa região causa, aos indivíduos afligidos, uma redução dos movimentos intervertebrais para, assim, diminuir a sensação de dor. O sistema de estabilização vertebral reage à dor ativamente para diminuir da zona de movimento intervertebral, através da ativação dos músculos ou pelo enrijecimento adaptativo da coluna vertebral ao longo do tempo, por exemplo, através da formação de osteófitos.

As causas mais comuns da lombalgia incluem má postura, desequilíbrio das estruturas passivas osteo-ligamentares, posturas hipocinéticas e atividade muscular, o que resulta na instabilidade do complexo lombo-pélvico e quadros dolorosos a ela relacionados. Além disso, quando há acometimentos relacionados à dor lombar, normalmente se observa inatividade, consequente da lesão que gera desequilíbrio entre comprimento, força, resistência e coordenação muscular ${ }^{8-10}$. $\mathrm{O}$ aumento das oscilações corporais também é observado nos indivíduos acometidos pela lombalgia ${ }^{11}$. Especificamente na canoagem, o equilíbrio corporal é uma importante capacidade física para a estabilidade na embarcação². Porém, o equilíbrio de canoístas em pé relacionado com as mudanças posturais, ao treinamento e à dor lombar pode ser prejudicado durante as práticas em canoagem e na saúde. Além disso, a dor lombar pode alterar a estabilidade corporal e consequentemente, interferir nos resultados do treinamento e na competição. Porém, estudos que relacionem os problemas musculoesqueléticos e a estabilidade corporal em atletas de alto rendimento são ainda necessários e merecem atenção dos pesquisadores. Diante dessas premissas, o presente estudo buscou identificar a influência da dor lombar sobre o equilíbrio corporal de atletas da seleção brasileira feminina de canoagem velocidade.

\section{PROCEDIMENTOS METODOLÓGICOS}

\section{Participantes}

Foram avaliadas $100 \%$ das atletas da seleção brasileira feminina de canoagem velocidade, composta por 11 atletas de alto nível do gênero feminino, representantes do Brasil nas provas mundiais da modalidade. As atletas foram divididas em $2 \mathrm{gru}-$ pos, ou seja, com e sem dores na região lombar. Os treinamentos das atletas são realizados diariamente de seis a oito horas, conforme relatos de Borges?

O grupo 1 foi composto pelas cinco atletas que afirmaram ter dor na região lombar em qualquer escala. A idade média das atletas do grupo com presença de dor lombar foi de 21,2 $\pm 5,26$ anos, massa corporal média de 63,84 $\pm 5,66 \mathrm{~kg}$, estatura média de 1,63 $\pm 0,03 \mathrm{~m}$, e tempo médio de prática na modalidade de 8,4 $\pm 3,05$ anos. O grupo 2 foi composto pelas seis atletas restantes, que afirmaram não ter dores na região lombar. A idade média das atletas foi de 20,5 \pm 4,72 anos, massa corporal média de 61,25 $\pm 5,93 \mathrm{~kg}$ e estatura média de 1,61 $\pm 0,05$ m e tempo de prática médio de 5,92 $\pm 1,86$ anos.

Após consentimento para participação da coleta de dados mediante assinatura do Termo de Consentimento Livre e Esclarecido, sob protocolo n 0095.0.243.000-07 do Comitê de Ética e Pesquisa da instituição de origem, foi preenchido um questionário para identificação de possíveis fatores de 
exclusão do estudo conforme Lemos et al. ${ }^{12}$. Tais critérios foram relacionados aos problemas musculoesqueléticos, como lesões osteo-mio-articulares, histórico de cirurgia da coluna vertebral ou fratura vertebral prévia a realização das coletas de dados, anormalidades estruturais da coluna vertebral (verificado através de histórico clínico), ter compressão do nervo ciático e dor irradiada ou presença de sintomas radiculares durante o exame físico, síndromes vestibulares, hipertensão e diabetes. Com base nesses critérios, nenhuma atleta foi impedida de prosseguir os procedimentos para coleta de dados.

\section{Instrumentos}

Para a identificação da dor lombar, as atletas receberam o questionário Brazil Rolan-Morris ${ }^{13}$ que relaciona a dor de forma qualitativa. A classificação da dor foi feita assim como ilustra a Tabela 1.

Tabela 1. Classificação da escala da dor com sua respectiva pontuação.

\begin{tabular}{lc}
\hline Escala da dor & Pontuação da escala de dor \\
\hline Dor quase insuportável & 5 \\
Dor muito forte & 4 \\
Dor forte & 3 \\
Dor moderada & 2 \\
Dor leve & 1 \\
Sem dor & 0 \\
\hline
\end{tabular}

Fonte: Nusbaum et al. ${ }^{13}$

De acordo com as respostas do questionário, as atletas foram classificadas em com e sem queixas de dor lombar, de acordo com a sensação da dor na última semana até o dia da coleta, na região lombar.

Para coleta dos dados específicos do equilí- brio, representados pelas variáveis amplitude de deslocamento do centro de pressão na direção ântero-posterior (COPap) e na direção médio-lateral (COPml), foi utilizado um estabilômetro AccuSway Plus, da marca AMTI (Advanced Mechanical Technology, INC), com utilização do software Balance Clinic para a compreensão dos dados. A posição dos pés das atletas para o reconhecimento da base de apoio utilizada foi feita pelo marcador do estabilômetro.

As atletas foram avaliadas em horários marcados, tendo um tempo de, aproximadamente, 10 minutos para familiarização com os equipamentos e explicações dos procedimentos por parte dos pesquisadores. Os equipamentos foram calibrados individualmente para cada atleta, respeitando as individualidades biológicas de cada uma como, por exemplo, a marcação da base de suporte.

Para a avaliação do equilíbrio corporal (ilustrado na Figura 1), as atletas foram instruídas a manter o olhar fixo em um ponto à frente (nas condições com o uso da visão, nas demais se utilizou uma venda para retirar a atuação do sistema visual) a uma distância de dois metros e permanecer o mais estático possível na posição anatômica de referência, em cima do estabilômetro, nas seis condições testadas:

1) com os pés distanciados na largura do quadril e olhos abertos (OABip);

2) em apoio unipodal esquerdo e olhos abertos (OAUE);

3) em apoio unipodal direito e olhos abertos (OAUD);

4) com os pés distanciados na largura do quadril e olhos fechados (com vendas) (OFBip);
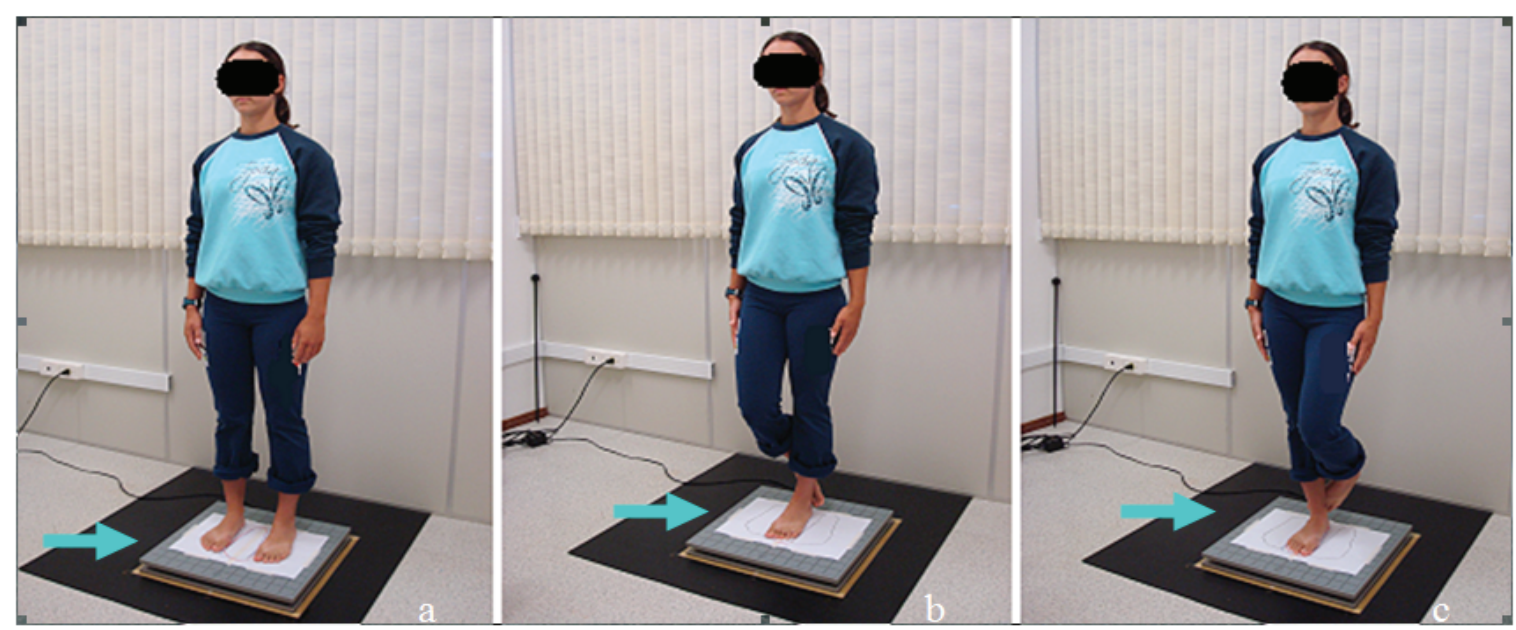

Figura 1. Demonstração da avaliação do equilíbrio corporal. (a) Bipodal, (b) unipodal esquerdo e (c) unipodal direito. A seta azul indica as marcações do posicionamento dos pés em cada condição. 
5) em apoio unipodal esquerdo e olhos fechados (com vendas) (OFUE);

6) em apoio unipodal direito e olhos fechados (com vendas) (OFUD).

O posicionamento dos pés, na primeira tentativa, foi demarcado com papel e caneta para manter o mesmo posicionamento nas tentativas posteriores a uma distância equivalente a largura do quadril (Figura 1). Foram realizadas três tentativas de 30 segundos para cada condição, totalizando 18 tentativas por atleta, sendo a frequência de aquisição dos sinais de $100 \mathrm{~Hz}$. Foi mantida uma temperatura constante da sala de coletas em $23^{\circ} \mathrm{C}$, para que as condições de teste estivessem com máximo de similaridade entre as atletas. Um filtro passa-baixa de $4^{a}$ ordem, próprio do programa Balance Clinic, foi utilizado para filtragem dos dados. A frequência de corte utilizada foi de $20 \mathrm{~Hz}$, conforme Lemos et al. ${ }^{12}$, porém, anteriormente à definição dessa frequiência, foi realizada uma análise do resíduo do sinal, que apresentou distribuição normal, o que indica que a suavização utilizada foi adequada.

\section{Análise estatistica}

Para a análise dos dados, foi utilizada a estatística descritiva por meio da média e desvio padrão. A normalidade dos dados foi verificada por meio do teste Shapiro-Wilk, que mostrou que os dados podem ser considerados como tendo uma distribuição normal. Para a comparação entre os grupos de atletas sem e com dor lombar, foi utilizado o teste t de Student para amostras independentes. O nível de significância utilizado para todos os testes foi de $5 \%$. Para as análises, foi utilizado o programa estatístico SPSS 11.5 for Windows.

\section{RESULTADOS}

Os resultados encontrados com relação às dores na região lombar estão ilustrados na Figura 2, sendo que $45,45 \%(n=5)$ relataram dores na região lombar (sendo classificadas como dor muito forte, dor forte, dor moderada e dor leve) e 54,55\% ( $\mathrm{n}=6$ ) não relataram a presença de dor.

Na Tabela 2, está ilustrado o nível de significância (p-valor) encontrado com as comparações das diferentes condições do equilíbrio corporal, na direção ântero-posterior (COPap), entre os grupos de atletas com e sem dor lombar.

Na Tabela 3, está ilustrado o nível de significância (p-valor) encontrado com as comparações das diferentes condições do equilíbrio corporal, na direção médio-lateral (COPml), entre os grupos de atletas com e sem dor lombar.

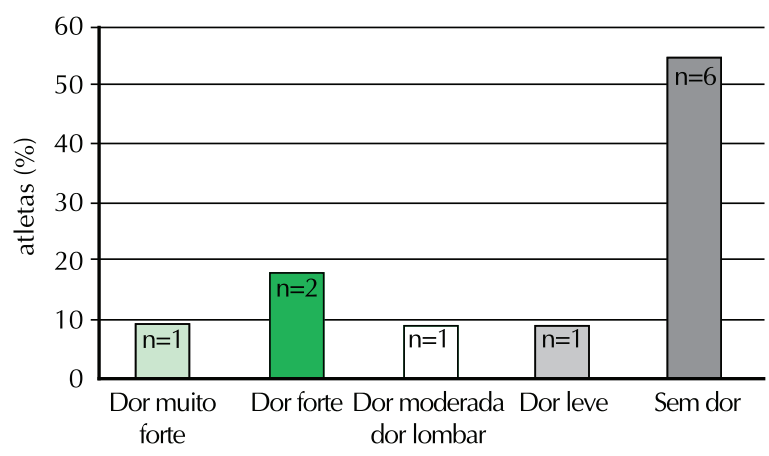

Figura 2. Distribuição das atletas conforme as queixas de dor na região lombar.

Tabela 2. Média e desvio padrão da amplitude de deslocamento no sentido ântero-posterior (COPap), nas diferentes condições, dos grupos com e sem dor lombar.

\begin{tabular}{lccc}
\hline Condições & $\begin{array}{c}\text { Com dor } \\
\text { lombar }\end{array}$ & $\begin{array}{c}\text { Sem dor } \\
\text { lombar }\end{array}$ & p-valor* \\
\hline OABip $(\mathrm{cm})$ & $2,08 \pm 0,63$ & $1,66 \pm 0,46$ & $0,034^{* *}$ \\
OAUD $(\mathrm{cm})$ & $4,36 \pm 1,35$ & $3,21 \pm 0,61$ & $0,003^{* *}$ \\
OAUE $(\mathrm{cm})$ & $4,00 \pm 0,95$ & $3,31 \pm 0,95$ & $0,046^{* *}$ \\
OFBip $(\mathrm{cm})$ & $2,70 \pm 0,41$ & $1,76 \pm 0,44$ & $<0,001^{* *}$ \\
OFUD $(\mathrm{cm})$ & $6,26 \pm 1,36$ & $6,63 \pm 2,10$ & 0,567 \\
OFUE $(\mathrm{cm})$ & $7,04 \pm 1,60$ & $7,68 \pm 2,61$ & 0,414 \\
\hline
\end{tabular}

* Probabilidade para o teste t de Student. **Valor menor que 0,05 indica diferença estatisticamente significativa entre os grupos sem e com dor lombar em cada condição testada

Tabela 3. Média e desvio padrão da amplitude de deslocamento do centro de pressão no sentido médio-lateral (COPml), nas diferentes condições, dos grupos com e sem dor lombar.

\begin{tabular}{lccc}
\hline Condições & $\begin{array}{c}\text { Com dor } \\
\text { lombar }\end{array}$ & $\begin{array}{c}\text { Sem dor } \\
\text { lombar }\end{array}$ & p-valor* \\
\hline OABip $(\mathrm{cm})$ & $1,31 \pm 0,40$ & $1,03 \pm 0,31$ & $0,029^{* *}$ \\
OAUD $(\mathrm{cm})$ & $2,73 \pm 0,41$ & $2,25 \pm 0,32$ & $0,001^{* *}$ \\
OAUE $(\mathrm{cm})$ & $2,75 \pm 0,49$ & $2,37 \pm 0,36$ & $0,017^{* *}$ \\
OFBip $(\mathrm{cm})$ & $1,70 \pm 0,53$ & $1,28 \pm 0,54$ & $0,031^{* *}$ \\
OFUD $(\mathrm{cm})$ & $4,61 \pm 0,59$ & $4,66 \pm 1,22$ & 0,901 \\
OFUE $(\mathrm{cm})$ & $4,74 \pm 0,43$ & $4,41 \pm 0,47$ & $0,043^{* *}$ \\
\hline
\end{tabular}

*Probabilidade para o teste $\mathrm{t}$ de Student. **Valor menor que 0,05 indica diferença estatisticamente significativa entre os grupos sem e com dor lombar em cada condição testada

Diante dos resultados expressos nas Tabelas 2 e 3, é possível observar que em todas as condições analisadas, com a utilização da visão, o equilíbrio das atletas com queixas de dor lombar foi significativamente menor quando comparadas às atletas sem queixas de dor lombar.

Com os olhos fechados, em apoio bipodal (OFBip), os resultados também foram estatisticamente diferentes, estando as atletas sem dor lombar 
com maiores estabilidades. Na condição unipodal, sem informação visual, não foram encontradas diferenças significativas entre os grupos, exceto na manutenção do equilíbrio com o membro inferior esquerdo (Tabela 3) para a amplitude de deslocamento na direção médio-lateral (COPml) em favor, mais uma vez, das atletas sem dor lombar.

\section{DISCUSSÃO}

O presente estudo buscou identificar a influência da dor lombar sobre o equilíbrio corporal de atletas da seleção brasileira feminina de canoagem velocidade.

Mesmo que a intensidade da dor possa ser interveniente na manutenção do equilíbrio corporal, o presente estudo limitou-se em classificar indivíduos com e sem a presença de queixas de dor. Além disso, as questões hormonais também podem ser consideradas como fatores intervenientes, uma vez que as mesmas não foram controladas no momento da coleta de dados.

Dentre os estudos investigados pelo presente trabalho, os acometimentos relacionados à dor lombar em atletas de canoagem foram encontrados apenas no estudo de Hensel et al. ${ }^{3}$, que reportou as lesões musculoesqueléticas, nas diferentes regiões corporais, apresentadas pelas atletas da seleção da brasileira feminina de canoagem. O estudo encontrou valores de 5,06 lesões por atleta a cada 1000 horas de atividade e, dentre estas lesões, as queixas de dor na região lombar foram prevalentes.

Estudos que relacionassem especificamente a canoagem e a manutenção da estabilidade estão começando a ser realizados, porém investigações que visem identificar as relações entre dor lombar e equilíbrio corporal ainda são escassas, principalmente em atletas.

Relacionando outras modalidades, Lemos et al. ${ }^{14}$ compararam praticantes de canoagem e praticantes de atletismo e natação, demonstrando um menor equilíbrio estático dos canoístas na posição anatômica de referência que os praticantes de outras atividades. Porém, investigações relacionadas ao esclarecimento destas diferenças não foram realizadas por outros estudos. Os mesmos autores expõem que as oscilações na direção médio-lateral podem apresentar-se diminuídas em decorrência da especificidade da modalidade de canoagem, que apresenta uma base de apoio médio-lateral, na posição sentada, diminuída em função das dimensões do caiaque, sendo estas transportadas para a posição em pé ${ }^{14}$.
Além disso, segundo Hamaoui et al. ${ }^{15}$, indivíduos com queixas de dor lombar apresentam aumento de magnitude do descolamento do centro de pressão, principalmente, na direção ântero-posterior, o que se associa com os resultados encontrados pelo presente estudo.

Para a permanência na água, o atleta deve controlar sua embarcação de forma a permanecer estável (com mínimo de oscilações verticais e médio-laterais) sem aumentar o atrito entre o barco e a água e sem despender força em direções que não auxiliem no rendimento. Além disso, a remada no caiaque é realizada com os membros superiores (MMSS), por meio de um remo de duas pás, com as quais o atleta rema dos dois lados do caiaque, numa trajetória simétrica ${ }^{3}$. A remada é considerada um movimento basicamente repetitivo e é realizado por um grande período de tempo com uma mecânica inapropriada e com sobrecarga repetida imposta a algumas regiões do corpo, o que, consequentemente, predispõe o atleta a lesões. Safran et al. ${ }^{16}$ citam que a exposição do atleta às forças aplicadas repetidamente e ao excesso de treinamento, leva a lesões por excesso de uso, sendo cerca de 30 a 50\% de todas as lesões esportivas.

Para Callagham e Mcgilli1 ${ }^{17}$, as cargas compressivas sobre a coluna lombar são aumentadas na posição sentada em relação à posição em pé. Para Hoogendoorn et al. ${ }^{18,}$ movimentação excessiva de rotação da coluna pode ser associada às causas de dor lombar. Fazendo uma relação com a modalidade de canoagem, pode-se dizer que, nesta modalidade, há um prejuízo no que se refere às cargas compressivas, fato que pode estar diretamente relacionado às queixas de dor lombar. Além disso, associações de rotação do tronco com flexão e extensão deste segmento durante a remada podem corroborar a incidência de queixas de dor devido ao uso excessivo e sobrecarga na região lombar.

McGill et al. ${ }^{19}$ afirmam que a região lombar é a que apresenta menor mobilidade articular, fazendo rotações de até 2 graus, enquanto que a torácica e cervical conseguem chegar a movimentos de até 10 graus. Esta consideração pode fazer com que haja susceptibilidade na região lombar no que tange aos movimentos de rotação. Logo, análises específicas com atletas de canoagem e suas relações com movimentos e lesões por meio de observação das demandas cinesiológicas, biomecânicas e fisiológicas do gesto esportivo ${ }^{3}$, ainda devem ser realizadas.

De forma geral, as queixas de dor na região lombar estão sendo evidenciadas por estudos que relacionam as instabilidades corporais ${ }^{20}$. As dife- 
renças na estabilidade corporal entre portadores de lombalgia e pessoas sem a patologia foram evidenciadas em estudos como os de Mok et al. ${ }^{20}$. Os autores avaliaram o equilíbrio corporal em posição bipodal, de 24 pacientes com lombalgia crônica e compararam com um grupo controle de 24 indivíduos sem dor lombar, com e sem o uso da visão. Os resultados foram semelhantes aos encontrados no presente estudo (Tabelas 2 e 3) e demonstraram as diferenças no equilíbrio entre os dois grupos, estando os acometidos pelas queixas de dor com menor estabilidade corporal.

Estas alterações podem ser em decorrência da incapacidade de utilização da estratégia do quadril no controle das oscilações corporais, para pessoas com problemas na região lombar, aumentando, consequentemente, os desequilíbrios e os valores do centro de pressão $0^{20}$. Além disso, a ausência ou menor utilização da estratégia do quadril sobrecarrega a utilização de outras estratégias de manutenção corporal em equilíbrio como, por exemplo, a do tornozelo.

O corpo em posição ereta é frequentemente comparado com um sistema de pêndulo, movendo-se pelo eixo dos tornozelos e, quando existe alguma alteração como, por exemplo, a lombalgia, que causa menor ação da região lombar na correção das instabilidades, aumenta-se o torque necessário para atuar motoramente na tarefa de trazer o centro de massa de volta para dentro da base de apoio, o que, consequentemente, aumenta os valores das variáveis de oscilação corporal ${ }^{21}$.

Harringe et al. ${ }^{22}$ afirmam que o controle do equilíbrio corporal é importante em esportes nos quais a técnica esportiva é imprescindível para se ter bons resultados. Como fatores intervenientes, a dor pode ser citada, uma vez que pode mudar a estratégia utilizada no controle postural. A coluna vertebral, em especial a musculatura lombar, age por informações eferentes para a manutenção do equilíbrio corporal ${ }^{23}$. Quando afetada por lesões, é menos efetiva e mais retardada no auxilio para correções de desequilíbrios ${ }^{24,25}$. Assim como os resultados encontrados no estudo de Lafond et al., ${ }^{11}$ que reportaram maior balanço postural nos indivíduos acometidos pela dor lombar, no caso do presente estudo, as atletas com queixas de dor na região lombar mostraram-se com as maiores instabilidades quando comparadas àquelas sem queixas (Tabelas 2 e 3).

Corroborando estes resultados, outras modalidades esportivas vêm sendo estudadas como, no caso, a ginástica. Harringe et $a .^{22}$ analisaram o equilíbrio corporal de ginastas suecas de alto nível esportivo, divididas em quatro grupos: sem lesão, com lombalgia, com dor no membro inferior e com múltiplas dores. Os maiores valores de oscilação corporal foram verificados para as atletas com dor lombar, estando as diferenças mais significativas nas condições mais complexas, ou seja, sem a utilização da visão e sobre uma plataforma instável (manipulando, respectivamente, os sistemas visual e proprioceptivo) ${ }^{24}$. Estes resultados assemelham-se aos aqui encontrados e são indicadores da influência da dor sobre as condições da estabilidade, tanto na canoagem quanto na ginástica.

No caso das avaliações com as condições em apoio unipodal, principalmente, sem a informação da visão, a dificuldade da tarefa para ambos os grupos, pode ser citada. De forma geral, além da diminuição da base de apoio que proporciona por si só maiores instabilidades ${ }^{26,27}$, há também a retirada de outra importante fonte de informação - a visual, fazendo com que o sistema vestibular e o somatossensorial sejam mais exigidos, aumentando a dificuldade da tarefa $22,28,29$. Estas considerações podem ser problemáticas para aqueles indivíduos com dor lombar, uma vez que, Lafond et al. ${ }^{11}$ indicaram alterações na função sensório-motora desses indivíduos, mostrando inabilidades para gerar respostas semelhantes aos indivíduos sem queixas.

\section{CONCLUSÃO}

De acordo com os resultados encontrados, o equilíbrio corporal das atletas femininas de canoagem velocidade de alto nível esportivo, participantes neste estudo, mostraram-se diferenciados na presença de dor lombar. As atletas com queixas mostraram-se com as maiores instabilidades.

\section{REFERÊNCIAS BIBLIOGRÁFICAS}

1. Nakamura FY, Borges TO, Sales OR, Cyrino ES, Kokubun E. Estimativa do custo energético e contribuição das diferentes vias metabólicas na canoagem de velocidade. Rev Bras Med Esporte 2004;10(2):70-7.

2. Lemos LFC, Pranke GI, Teixeira CS. Metodologia para o aprendizado da canoagem. EFDeportes 2007;12(114).

3. Hensel P, Perroni MG, Leal Júnior ECP. Lesões musculoesqueléticas na temporada de 2006 em atletas da seleção brasileira feminina principal de canoagem velocidade. Acta Ortop Bras 2008,16:4:233-7.

4. Medrado MA. Gestão do conhecimento e ergonomia: fatores biomecânicos na atividade de soldagem de peças. 2007 [Dissertação de Mestrado - Programa de pós-graduação em Engenharia da Produção]. Florianópolis (SC): Universidade Federal de Santa Catarina; 2007. 
5. Leveille SG, Zhang Y, Mcmullen W, Kelly-Hayes M, Felson DT. Sex differences in musculoskeletal pain in older adults. Pain 2005;116:332-8.

6. Andersen LA, Wedderkopp N, Leboeuf-yde C. Association between back pain and physical fitness in adolescents. Spine 2006;31:1740-4.

7. Borges TO. Canoagem Velocidade: Dinâmica das cargas de treinamento no macrociclo e a dinâmica da alteração de marcadores funcionais externos. [Dissertação de Mestrado - Programa de pós-graduação em Educação Física]. Campinas (SP): Universidade Estadual de Campinas; 2008.

8. Panjabi MM. Clinical spinal instability and low-back pain. J Electromyogr Kinesiol 2003;13:371-9.

9. Vogt L. Neuromuscular control of walking with chronic low back pain. Manual Ther 2003; 8: 21 - 28.

10. Renkawitz TD, Boluki DJ, Grifka J. The association of low back pain, neuromuscular imbalance, and trunk extension strength in athletes. Spine 2006;6:673-83.

11. Lafond D, Champagne A, Descarreaux M, Dubois JD, Prado JM, Duarte M. Postural control during prolonged standing in persons with chronic low back pain. Gait Post 2009; 29(3):421-7.

12. Lemos LFC, Teixeira CS, David AC, Mota CB. Equilíbrio corporal de atletas da seleção brasileira feminina de canoagem velocidade. Braz J Biomech 2009;10(18):22-8.

13. Nusbaum L, Natour J, Ferraz MB, Goldenberg J. Translation, adaptation and validation of the Roland-Morris questionnaire - Brazil Rolan-Morris. Braz J Med Biol Res 2001; 34(2):203-10.

14. Lemos LFC, Mann L, Pranke GI, Teixeira CS, Rossi AG, Mota CB. Investigação do equilíbrio estático em praticantes de canoagem velocidade. Anais eletronicos do XII Congresso Brasileiro de Biomecânica. Rio Claro: UNESP; 2007.

15. Hamaoui A, Le Bozec S, Poupard L, Bouisset S. Does postural chain muscular stiffness reduce postural steadiness in a sitting posture. Gait Post 2007;25:199-204.

16. Safran MR, Mckeag DB, Camp SPV. Manual de medicina esportiva. São Paulo: Manole; 2002.

17. Callaghan JP, McGill SM. Intervertebral disc herniation: Studies on a porcine model exposed to highly repetitive flexion/extension motion with compressive force. Clin Biom 2001;16(1):28-37.

18. Hoogendoorn WE, Bongers PM, de Vet HC, Douwes M, Koes BW, Miedema MC, et al. Flexion and rotation of the trunk and lifting at work are risk factors for low back pain: results of a prospective cohort study. Spine 2000;25:3087-92.

19. McGill SM, Yingling VR, Peach JP. Tree-dimensional kinematics and trunk muscle myoelectric activity in elderly spine - a database compared to young people. Clin Biomechanics 1999;14:389-95.

20. Mok NW, Brauer SG, Hodges PW. Hip Strategy for Balance Control in Quiet Standing Is Reduced in People With Low Back Pain Spine. 2004;29(6):107-12.
21. Hue O, Simoneau M, Marcotte J, Berrigan F, Doré J, Marceau P, et al. Body Weight is a strong predictor of postural stability. Gait Post 2007;26:32-8.

22. Harringe ML, Halvorsen K, Renström P, Werner S. Postural control measured as the center of pressure excursion in young female gymnasts with low back pain or lower extremity injury. Gait Post 2008;28:38-45.

23. Holm S, Indahl A, Solomonow M. Sensoriomotor control of the spine. J Electromyogr Kinesiol 2002;12:219-34.

24. Radebold A, Cholewicki J, Polzhofer GK, Greene HS. Impaired Postural Control of the Lumbar Spine Is Associated With Delayed Muscle Response Times in Patients With Chronic Idiopathic Low Back Pain. Spine 2001;26(7):724-30.

25. Leinonen V, Määttä S, Taimela S, Herno A, Kankaanpää M, Partanen J, et al. Impaired lumbar position sense in association with postural stability and motor and somatosensory evoked potential findings in lumbar spinal stenosis. Spine 2002;27:975-83.

26. Latash ML, Ferreira SS, Wieczorek AS, Duarte M. Movement sway: changes in postural sway during voluntary shifts of the center of pressure. Exp Brain Res 2003;150: 314-24.

27. Mochizuki L, Duarte M, Amadio AC, Zatsiorsky VM, Latash ML. Changes in Postural Sway and Its Fractions in Conditions of Postural Instability. JAB 2006;22(1):51-60.

28. Baydal-Bertomeu JM, Guillem RB, Soler-Gracia C, Moya MFP, Prat JM, Guzmán RB. Determinación de los patrones de comportamiento postural en población sana española. Acta Otorinolaringol Esp 2004;55:260-9.

29. Patel M, Fransson PA, Lush D, Gomez S. The effect of foam surface properties on postural stability assessment while standing. Gait Post 2008:1-8. 